\title{
EXPECTATION IDENTITIES OF GENERALIZED RECORD VALUES FROM NEW WEIBULL-PARETO DISTRIBUTION AND ITS CHARACTERIZATION
}

\author{
M. A. R. KHAN ${ }^{1}$, R. U. KHAN $^{1}$ and M. A. KHAN ${ }^{2}$ \\ ${ }^{1}$ Department of Statistics and Operations Research \\ Aligarh Muslim University \\ Aligarh-202002 \\ India \\ e-mail: aruke@rediffmail.com \\ 2 Jaipuria Institute of Management \\ Lucknow-226010 \\ India
}

\begin{abstract}
In this paper, we consider New Weibull-Pareto distribution, was recently introduced by Nasiru and Luguterah [16]. Exact expressions as well as some recurrence relations for single and product moments of generalized upper record values ( $k$-th upper record values) are derived. Further, various deductions and particular cases are discussed. At the end, the characterization results based on conditional expectation and recurrence relations are presented and some computational work also carried out.
\end{abstract}

2010 Mathematics Subject Classification: 62G30, 62E10, 60E05.

Keywords and phrases: order statistics, generalized record values, $k$-th record values, New

Weibull-Pareto distribution, single moments, product moments, recurrence relations, conditional expectation and characterization.

Received October 25, 2017

(ㄷ) 2017 Scientific Advances Publishers 


\section{Introduction}

The statistical study of record values in a sequence of independent and identically distributed (iid) continuous random variables was first carried out by Chandler [2]. Dziubdziela and Kopociński [3] have generalized the concept of record values of Chandler [2] by random variables of a more generalized nature and called them the $k$-th record values. Later, Minimol and Thomas [14] call the record values defined by Dziubdziela and Kopociński [3] also as the generalized record values, since the $r$-th member of the sequence of the ordinary record values is also known as the $r$-th record value. Setting $k=1$, we obtain ordinary record statistics.

Applications of $k$-th record values can be found in the literature, for instance, see the examples cited in Kamps [9] or Danielak and Raqab [4] in reliability theory. Suppose that a technical system or piece of equipment is subject to shocks, e.g., peaks of voltages. If the shocks are viewed as realizations of an iid sequence, then the model of ordinary records is adequate. If it is not the records themselves, but second or third values are of special interest, then the model of $k$-th record values is adequate. When record values themselves are viewed as outliers, then the second or third largest values are of special interest. Record statistics are applied in estimating strength of materials, predicting natural disasters, sport achievements, etc. For statistical inference based on ordinary records, serious difficulties arise if expected values of inter arrival time of records is infinite and occurrences of records are very rare in practice. This problem is avoided once we consider the model of $k$-th record statistics.

Let $\left\{X_{n}, n \geq 1\right\}$ be a sequence of iid random variables with distribution function $(d f) F(x)$ and probability density function ( $p d f) f(x)$. The $j$-th order statistic of a sample $X_{1}, X_{2}, \ldots, X_{n}$ is 
denoted by $X_{j: n}$. For a fixed positive integer $k$, we define the sequence $\left\{U_{n}^{(k)}, n \geq 1\right\}$ of $k$-th upper record times of $\left\{X_{n}, n \geq 1\right\}$ as follows:

$$
\begin{aligned}
& U_{1}^{(k)}=1, \\
& U_{n+1}^{(k)}=\min \left\{j>U_{n}^{(k)}: X_{j: j+k-1}>X_{U_{n}^{(k)}: U_{n}^{(k)}+k-1}\right\} .
\end{aligned}
$$

The sequence $\left\{Y_{n}^{(k)}, n \geq 1\right\}$, where $Y_{n}^{(k)}=X_{U_{n}^{(k)}}$ is called the sequence of $k$-th upper record values or generalized upper record values of $\left\{X_{n}, n \geq 1\right\}$. Note that for $k=1$, we write $Y_{n}^{(1)}=X_{U_{n}}, n \geq 1$, which are the upper record values of $\left\{X_{n}, n \geq 1\right\}$ as defined in Ahsanullah [1]. Moreover, we see that $Y_{0}^{(k)}=0$ and $Y_{1}^{(k)}=\min \left(X_{1}, X_{2}, \ldots, X_{n}\right)=X_{1: k}$.

The $p d f$ of $Y_{n}^{(k)}$ and the joint $p d f$ of $Y_{m}^{(k)}$ and $Y_{n}^{(k)}$ are given by (Dziubdziela and Kopociński [3]; Grudzień [6])

$$
f_{Y_{n}^{(k)}}(x)=\frac{k^{n}}{(n-1) !}[-\ln \bar{F}(x)]^{n-1}[\bar{F}(x)]^{k-1} f(x), \quad n \geq 1,
$$

and

$$
\begin{gathered}
f_{Y_{m}^{(k)}, Y_{n}^{(k)}}(x, y)=\frac{k^{n}}{(m-1) !(n-m-1) !}[-\ln \bar{F}(x)]^{m-1} \frac{f(x)}{\bar{F}(x)} \\
\times[\ln \bar{F}(x)-\ln \bar{F}(y)]^{n-m-1}[\bar{F}(y)]^{k-1} f(y), \\
x<y, \quad 1 \leq m<n, \quad n \geq 2,
\end{gathered}
$$

where

$$
\bar{F}(x)=1-F(x)
$$

For some recent developments on generalized upper record values with special reference to those arising from exponential, Gumble, Pareto, generalized Pareto, Burr, Weibull, Gompertz, Makeham, exponential- 
Weibull, modified Weibull, and additive Weibull distributions, see Grudzień and Szynal [7], Pawlas and Szynal [17, 18, 19], Minimol and Thomas [14, 15], Khan et al. [13], Khan and Khan [11], and Khan et al. [12], respectively. In this work, we mainly focus on the study of generalized upper record values arising from the New Weibull-Pareto distribution.

A random variable $X$ is said to have a New Weibull-Pareto distribution (Nasiru and Luguterah [16]), if its $p d f$ is of the form

$$
f(x)=\frac{\alpha \beta}{\theta}(x / \theta)^{\beta-1} e^{-\alpha(x / \theta)^{\beta}}, \quad 0<x<\infty, \quad \alpha>0, \beta, \theta>0,
$$

with the corresponding $d f$

$$
F(x)=1-e^{-\alpha(x / \theta)^{\beta}}, \quad 0<x<\infty, \quad \alpha>0, \beta, \theta>0 .
$$

It is easy to see that

$$
f(x)=\frac{\alpha \beta}{\theta^{\beta}} x^{\beta-1} \bar{F}(x) .
$$

Nasiru and Luguterah [16] pointed out the New Weibull-Pareto distribution provides greater flexibility in the modelling of lifetime data. The results, on real life data compared with other known distributions, revealed that this distribution provides a better fit for modelling real life data.

The main properties of the New Weibull-Pareto distribution are as follows:

(i) If $\beta=1$, the failure rate is constant. This makes New WeibullPareto distribution suitable for modelling system or components with constant failure rate.

(ii) If $\beta>1$, the hazard rate is an increasing function of $x$, which makes the New Weibull-Pareto distribution suitable for modelling components that wears faster with time. 
(iii) If $\beta<1$, the failure rate is a decreasing function of $x$, which makes the New Weibull-Pareto distribution suitable for modelling components that wears slower with time.

The relation in (1.5) will be exploited in this study to derive explicit expressions and some recurrence relations for the moments of generalized upper record values from the New Weibull-Pareto distribution.

\section{Relations for Single Moments}

Here we will derive the exact expressions and recurrence relations for single moments of generalized upper record values from the New Weibull-Pareto distribution. First we describe the exact expression for single moments of generalized upper record values in the following theorem:

Theorem 2.1. For the distribution given in (1.4). Fix a positive integer $k \geq 1$, for $n \geq 1, n \geq k$ and $j=0,1, \ldots$

$$
E\left(Y_{n}^{(k)}\right)^{j}=\frac{\theta^{j} \Gamma[n+(j / \beta)]}{(n-1) !(\alpha k)^{j / \beta}} .
$$

Proof. From (1.1) and (1.5), we have

$$
E\left(Y_{n}^{(k)}\right)^{j}=\frac{\alpha \beta k^{n}}{(n-1) ! \theta^{\beta}} \int_{0}^{\infty} x^{j+\beta-1}[-\ln \bar{F}(x)]^{n-1}[\bar{F}(x)]^{k} d x
$$

Setting $t=-\ln \bar{F}(x)$ in (2.2), we find that

$$
E\left(Y_{n}^{(k)}\right)^{j}=\frac{\theta^{j} k^{n}}{(n-1) ! \alpha^{j / \beta}} \int_{0}^{\infty} t^{n+(j / \beta)-1} e^{-t k} d t
$$

and hence the result given in (2.1).

Corollary 2.1. The exact expression for single moments of upper record values from the New Weibull-Pareto distribution has the form 


$$
E\left(Y_{n}^{(1)}\right)^{j}=E\left(X_{U_{n}}^{j}\right)=\frac{\theta^{j} \Gamma[n+(j / \beta)]}{(n-1) ! \alpha^{j / \beta}} .
$$

Numerical computations for the first four moments of upper record values from New Weibull-Pareto distribution for arbitrary chosen values of $\alpha, \beta, \theta$ and various sample size $n=1,2, \ldots, 5$ are given in Table 2.1.

Table 2.1. First four moments of upper record values

\begin{tabular}{|c|c|c|c|c|c|c|c|c|}
\hline \multirow{2}{*}{$n$} & \multicolumn{5}{|c|}{$\alpha=1, \beta=2, \theta=1$} & \multicolumn{4}{c|}{$\alpha=2, \beta=2, \theta=1$} \\
\cline { 2 - 9 } & $E(X)$ & $E\left(X^{2}\right)$ & $E\left(X^{3}\right)$ & $E\left(X^{4}\right)$ & $E(X)$ & $E\left(X^{2}\right)$ & $E\left(X^{3}\right)$ & $E\left(X^{4}\right)$ \\
\hline 1 & 0.88623 & 1.000 & 1.32934 & 2.000 & 0.62666 & 0.500 & 0.46999 & 0.500 \\
\hline 2 & 1.32934 & 2.000 & 3.32335 & 6.000 & 0.93999 & 1.000 & 1.17498 & 1.500 \\
\hline 3 & 1.66168 & 3.000 & 5.81586 & 12.000 & 1.17498 & 1.500 & 2.05622 & 3.000 \\
\hline 4 & 1.93862 & 4.000 & 8.72380 & 20.000 & 1.37081 & 2.000 & 3.08433 & 5.000 \\
\hline 5 & 2.18095 & 5.000 & 11.99522 & 30.000 & 1.54216 & 2.500 & 4.24095 & 7.500 \\
\hline & & $\alpha=1, \beta=2, \theta=2$ & & & $\alpha=2, \beta=2, \theta=2$ & \\
\cline { 2 - 9 } & $E(X)$ & $E\left(X^{2}\right)$ & $E\left(X^{3}\right)$ & $E\left(X^{4}\right)$ & $E(X)$ & $E\left(X^{2}\right)$ & $E\left(X^{3}\right)$ & $E\left(X^{4}\right)$ \\
\hline 1 & 1.77245 & 4.000 & 10.63472 & 32.000 & 1.25331 & 2.000 & 3.75994 & 8.000 \\
\hline 2 & 2.65868 & 8.000 & 26.58681 & 96.000 & 1.87997 & 4.000 & 9.39986 & 24.000 \\
\hline 3 & 3.32335 & 12.000 & 46.52691 & 192.000 & 2.34996 & 6.000 & 16.44975 & 48.000 \\
\hline 4 & 3.87724 & 16.000 & 69.79037 & 320.000 & 2.74163 & 8.000 & 24.67462 & 80.000 \\
\hline 5 & 4.36190 & 20.000 & 95.96176 & 480.000 & 3.08433 & 10.000 & 33.92761 & 120.000 \\
\hline
\end{tabular}

Now, we obtain the recurrence relations for single moments of generalized upper record values from the New Weibull-Pareto distribution in the following theorem:

Theorem 2.2. For the distribution given in (1.4). Fix a positive integer $k \geq 1$, for $n \geq 1, n \geq k$ and $j=0,1, \ldots$

$$
E\left(Y_{n}^{(k)}\right)^{j+\beta}=E\left(Y_{n-1}^{(k)}\right)^{j+\beta}+\frac{(j+\beta) \theta^{\beta}}{\alpha \beta k} E\left(Y_{n}^{(k)}\right)^{j} .
$$


Proof. From (1.1) and (1.5), for $n \geq 1$ and $j=0,1, \ldots$, we have

$$
E\left(Y_{n}^{(k)}\right)^{j}=\frac{\alpha \beta k^{n}}{(n-1) ! \theta^{\beta}} \int_{0}^{\infty} x^{j+\beta-1}[-\ln \bar{F}(x)]^{n-1}[\bar{F}(x)]^{k} d x
$$

Now (2.4) can be seen by noting that in view of Khan et al. [12]

$$
E\left(Y_{n}^{(k)}\right)^{j}-E\left(Y_{n-1}^{(k)}\right)^{j}=\frac{j k^{n-1}}{(n-1) !} \int_{0}^{\infty} x^{j-1}[-\ln \bar{F}(x)]^{n-1}[\bar{F}(x)]^{k} d x
$$

Remark 2.1. (i) Setting $\theta=1$ in (2.4), we get the relation for single moments of generalized upper record values from the Weibull distribution, which verify the results obtained by Pawlas and Szynal [17].

(ii) Putting $\alpha=1, \beta=1, \theta=1$ in (2.4), we deduce the recurrence relation for single moments of $k$-th upper record values from the exponential distribution, established by Pawlas and Szynal [18].

(iii) Putting $\beta=2, \theta=1$ in (2.4), the recurrence relation for single moments of $k$-th upper record values is deduced for the Rayleigh distribution as obtained by Khan et al. [13].

Corollary 2.2. The recurrence relation for single moments of upper record values from the New Weibull-Pareto distribution has the form

$$
E X_{U_{n}}^{j+\beta}=E X_{U_{n-1}}^{j+\beta}+\frac{(j+\beta) \theta^{\beta}}{\alpha \beta} E X_{U_{n}}^{j}
$$

\section{Relations for Product Moments}

This section contains the explicit expressions and recurrence relations for product moments of generalized upper record values from the New Weibull-Pareto distribution. We shall first establish the explicit expression for the product moments of generalized upper record values in the following theorem:

Theorem 3.1. For the distribution given in (1.2). Fix a positive integer $k \geq 1$, for $1 \leq m \leq n-2$ and $i, j=0,1, \ldots$ 


$$
\begin{aligned}
E\left[\left(Y_{m}^{(k)}\right)^{i}\left(Y_{n}^{(k)}\right)^{j}\right]= & \frac{\theta^{i+j} k^{n}}{\alpha^{(i+j) / \beta}(m-1) !(n-m-1) !} \sum_{u=0}^{n-m-1} \frac{(-1)^{n-m-u-1}}{[n+(i / \beta)-u-1]} \\
& \times\left(\begin{array}{c}
n-m-1 \\
u
\end{array}\right) \frac{\Gamma[n+(i+j) / \beta]}{k^{n+(i+j) / \beta}}
\end{aligned}
$$

Proof. From (1.2), we have

$$
\begin{aligned}
E\left[\left(Y_{m}^{(k)}\right)^{i}\left(Y_{n}^{(k)}\right)^{j}\right]= & \frac{k^{n}}{(m-1) !(n-m-1) !} \int_{0}^{\infty} \int_{0}^{y} x^{i} y^{j}[-\ln \bar{F}(x)]^{m-1} \frac{f(x)}{\bar{F}(x)} \\
& \times[\ln \bar{F}(x)-\ln \bar{F}(y)]^{n-m-1}[\bar{F}(y)]^{k-1} f(y) d x d y .
\end{aligned}
$$

On expanding $[\ln \bar{F}(x)-\ln \bar{F}(y)]^{n-m-1}$ binomially in (3.2), we get

$$
\begin{aligned}
E\left[\left(Y_{m}^{(k)}\right)^{i}\left(Y_{n}^{(k)}\right)^{j}\right]= & \frac{k^{n}}{(m-1) !(n-m-1) !} \sum_{u=0}^{n-m-1}(-1)^{n-m-u-1}\left(\begin{array}{c}
n-m-1 \\
u
\end{array}\right) \\
& \times \int_{0}^{\infty} y^{j}[-\ln \bar{F}(y)]^{u}[\bar{F}(y)]^{k-1} f(y) I(y) d y
\end{aligned}
$$

where

$$
I(y)=\int_{0}^{y} x^{i}[-\ln \bar{F}(x)]^{n-u-2} \frac{f(x)}{\bar{F}(x)} d x .
$$

By setting $t=-\ln \bar{F}(x)$ in (3.4), we obtain

$$
\begin{aligned}
I(y) & =\frac{\theta^{i}}{\alpha^{i / \beta}} \int_{0}^{-\ln \bar{F}(y)} t^{n+(i / \beta)-u-2} d t \\
& =\frac{\theta^{i}}{\alpha^{i / \beta}[n+(i / \beta)-u-1]}[-\ln F(y)]^{n+(i / \beta)-u-1} .
\end{aligned}
$$

On substituting the above expression of $I(y)$ in (3.3), we find that 


$$
\begin{aligned}
E\left[\left(Y_{m}^{(k)}\right)^{i}\left(Y_{n}^{(k)}\right)^{j}\right]= & \frac{\theta^{i} k^{n}}{\alpha^{i / \beta}(m-1) !(n-m-1) !} \sum_{u=0}^{n-m-1} \frac{(-1)^{n-m-u-1}}{[n+(i / \beta)-u-1]}\left(\begin{array}{c}
n-m-1 \\
u
\end{array}\right) \\
& \times \int_{0}^{\infty} y^{j}[-\ln F(y)]^{n+(i / \beta)-1}[\bar{F}(y)]^{k-1} f(y) d y
\end{aligned}
$$

Again by setting $z=-\ln \bar{F}(y)$ in (3.5) and simplifying the resulting expression, we get

$$
E\left[\left(Y_{m}^{(k)}\right)^{i}\left(Y_{n}^{(k)}\right)^{j}\right]=A \int_{0}^{\infty} z^{[n+(i+j) / \beta]-1} e^{-k z} d z
$$

where

$$
A=\frac{\theta^{i+j} k^{n}}{\alpha^{(i+j) / \beta}(m-1) !(n-m-1) !} \sum_{u=0}^{n-m-1} \frac{(-1)^{n-m-u-1}}{[n+(i / \beta)-u-1]}\left(\begin{array}{c}
n-m-1 \\
u
\end{array}\right),
$$

and hence the result given in (3.1).

Identity 3.1. For $1 \leq m<n$,

$$
\sum_{u=0}^{n-m-1} \frac{(-1)^{n-m-u-1}}{(n-u-1)}\left(\begin{array}{c}
n-m-1 \\
u
\end{array}\right)=\frac{(m-1) !(n-m-1) !}{(n-1) !} .
$$

Proof. Putting $i=j=0$ in (3.1), we get the required result.

Remark 3.1. At $i=0$ in (3.1), we have

$$
\begin{gathered}
E\left(Y_{n}^{(k)}\right)^{j}=\frac{\theta^{j} k^{n}}{\alpha^{j / \beta}(m-1) !(n-m-1) !} \sum_{u=0}^{n-m-1} \frac{(-1)^{n-m-u-1}}{(n-u-1)}\left(\begin{array}{c}
n-m-1 \\
u
\end{array}\right) \\
\times \frac{\Gamma[n+(j / \beta)]}{k^{n+(j / \beta)}}
\end{gathered}
$$

Making use of (3.6) in (3.7), we find that

$$
E\left(Y_{n}^{(k)}\right)^{j}=\frac{\theta^{j} k^{n}}{(n-1) ! \alpha^{j / \beta}} \frac{\Gamma[n+(j / \beta)]}{k^{n+(j / \beta)}},
$$


which is the exact expression for single moments from the New WeibullPareto distribution, as obtained in (2.1).

Corollary 3.1. The exact expression for product moments of upper record values from the New Weibull-Pareto distribution has the form

$$
\begin{aligned}
E\left[\left(Y_{m}^{(1)}\right)^{i}\left(Y_{n}^{(1)}\right)^{j}\right]= & E\left(X_{U_{m}}^{i} X_{U_{n}}^{j}\right)=\frac{\theta^{i+j}}{\alpha^{(i+j) / \beta}(m-1) !(n-m-1) !} \\
& \times \sum_{u=0}^{n-m-1} \frac{(-1)^{n-m-u-1}}{[n+(i / \beta)-u-1]}\left(\begin{array}{c}
n-m-1 \\
u
\end{array}\right) \Gamma[n+(i+j) / \beta] .
\end{aligned}
$$

The following theorem gives the recurrence relations for product moments of generalized upper record values.

Theorem 3.2. For the distribution given in (1.4) and $m \geq 1, m \geq k$ and $i, j=0,1, \ldots$

$$
E\left[\left(Y_{m}^{(k)}\right)^{i}\left(Y_{m+1}^{(k)}\right)^{j+\beta}\right]=E\left(Y_{m}^{(k)}\right)^{i+j+\beta}+\frac{\theta^{\beta}(j+\beta)}{\alpha \beta k} E\left[\left(Y_{m}^{(k)}\right)^{i}\left(Y_{m+1}^{(k)}\right)^{j}\right]
$$

and for $1 \leq m \leq n-2, i, j=0,1, \ldots$

$$
E\left[\left(Y_{m}^{(k)}\right)^{i}\left(Y_{n}^{(k)}\right)^{j+\beta}\right]=E\left[\left(Y_{m}^{(k)}\right)^{i}\left(Y_{n-1}^{(k)}\right)^{j+\beta}\right]+\frac{\theta^{\beta}(j+\beta)}{\alpha \beta k} E\left[\left(Y_{m}^{(k)}\right)^{i}\left(Y_{n}^{(k)}\right)^{j}\right]
$$

Proof. From (1.2) and (1.5), we have

$$
\begin{aligned}
E\left[\left(Y_{m}^{(k)}\right)^{i}\left(Y_{n}^{(k)}\right)^{j}\right]= & \frac{\alpha \beta k^{n}}{(m-1) !(n-m-1) ! \theta^{\beta}} \int_{0}^{\infty} \int_{x}^{\infty} x^{i} y^{j+\beta-1}[-\ln \bar{F}(x)]^{m-1} \frac{f(x)}{\bar{F}(x)} \\
& \times[\ln \bar{F}(x)-\ln \bar{F}(y)]^{n-m-1}[\bar{F}(y)]^{k} d y d x
\end{aligned}
$$

(3.10) can be proved in view of Khan et al. [12] by noting that

$$
E\left[\left(Y_{m}^{(k)}\right)^{i}\left(Y_{n}^{(k)}\right)^{j}\right]-E\left[\left(Y_{m}^{(k)}\right)^{i}\left(Y_{n-1}^{(k)}\right)^{j}\right]
$$




$$
\begin{aligned}
= & \frac{j k^{n-1}}{(m-1) !(n-m-1) !} \int_{0}^{\infty} \int_{x}^{\infty} x^{i} y^{j-1}[-\ln \bar{F}(x)]^{m-1} \frac{f(x)}{\bar{F}(x)} \\
& \times[\ln \bar{F}(x)-\ln \bar{F}(y)]^{n-m-1}[\bar{F}(y)]^{k} d y d x .
\end{aligned}
$$

Proceeding in a similar manner for the case $n=m+1$, the recurrence relation given in (3.8) can easily be established.

One can also note that Theorem 2.2 can be deduced from Theorem 3.2 by putting $i=0$.

Remark 3.2. (i) Setting $\theta=1$ in (3.9), we get the recurrence relation for product moments of generalized upper record values from the Weibull distribution, which verify the results obtained by Pawlas and Szynal [17].

(ii) Putting $\alpha=1, \beta=1, \theta=1$ in (3.9), we deduce the recurrence relation for product moments of generalized upper record values from the exponential distribution, established by Pawlas and Szynal [18].

(iv) Putting $\beta=2, \theta=1$ in (3.9), the recurrence relation for product moments of generalized upper record values is deduced for the Rayleigh distribution as obtained by Khan et al. [13].

Corollary 3.2. The recurrence relations for product moments of upper record values from New Weibull-Pareto distribution has the form

$$
E\left(X_{U_{m}}^{i} X_{U_{n}}^{j+\beta}\right)=E\left(X_{U_{m}}^{i} X_{U_{n-1}}^{j+\beta}\right)+\frac{\theta^{\beta}(j+\beta)}{\alpha \beta} E\left(X_{U_{m}}^{i} X_{U_{n}}^{j}\right)
$$

\section{Characterizations}

Theorem 4.1. Fix a positive integer $k \geq 1$ and let $j$ be a non-negative integer, a necessary and sufficient condition for a random variable $X$ to be distributed with $f(x)$ given by (1.3) is that

$$
E\left(Y_{n}^{(k)}\right)^{j+\beta}=E\left(Y_{n-1}^{(k)}\right)^{j+\beta}+\frac{(j+\beta) \theta^{\beta}}{\alpha \beta k} E\left(Y_{n}^{(k)}\right)^{j}
$$

for $n=1,2, \ldots$ and $n \geq k$. 
Proof. The necessary part follows immediately from (2.5). On the other hand if the recurrence relation in (4.1) is satisfied, then on using (1.1), we have

$$
\begin{aligned}
& \frac{k^{n}}{(n-1) !} \int_{0}^{\infty} x^{j+\beta}[-\ln \bar{F}(x)]^{n-1}[\bar{F}(x)]^{k-1} f(x) d x \\
& \quad=\frac{k^{n-1}}{(n-2) !} \int_{0}^{\infty} x^{j+\beta}[-\ln \bar{F}(x)]^{n-2}[\bar{F}(x)]^{k-1} f(x) d x \\
& \quad+\frac{(j+\beta) \theta^{\beta} k^{n-1}}{\alpha \beta(n-1) !} \int_{0}^{\infty} x^{j}[-\ln \bar{F}(x)]^{n-1}[\bar{F}(x)]^{k-1} f(x) d x
\end{aligned}
$$

Integrating the left hand side in (4.2) by parts and simplifying the resulting expression, we find that

$$
\frac{(j+\beta) k^{n-1}}{(n-1) !} \int_{0}^{\infty} x^{j}[-\ln \bar{F}(x)]^{n-1}[\bar{F}(x)]^{k-1}\left\{\frac{\theta^{\beta}}{\alpha \beta} f(x)-x^{\beta-1} \bar{F}(x)\right\} d x=0 .
$$

Now applying a generalization of the Müntz-Szász Theorem (see, for example, Hwang and Lin [8]) to (4.3), we obtain

$$
f(x)=\frac{\alpha \beta}{\theta^{\beta}} x^{\beta-1} \bar{F}(x),
$$

which proves that $f(x)$ has the form as in (1.5).

Corollary 4.1. Under the assumptions of Theorem 4.1 with $j=0$, the following equation:

$$
E\left(Y_{n}^{(k)}\right)^{\beta}=E\left(Y_{n-1}^{(k)}\right)^{\beta}+\frac{\beta \theta^{\beta}}{\alpha \beta k}, n=1,2, \ldots
$$

characterize the New Weibull-Pareto distribution.

Remark 4.1. If $k=1$, we obtain the following characterization of the New Weibull-Pareto distribution: 


$$
E X_{U_{n}}^{\beta}=E X_{U_{n-1}}^{\beta}+\frac{\beta \theta^{\beta}}{\alpha \beta}, \quad n=1,2, \ldots
$$

Theorem 4.2. Let $X$ be a non-negative random variable having an absolutely continuous if $F(x)$ with $F(0)=0$ and $0 \leq F(x) \leq 1$ for all $x>0$, then

$$
E\left[\xi\left(Y_{n}^{(k)}\right) \mid\left(Y_{l}^{(k)}\right)=x\right]=e^{-\alpha(x / \theta)^{\beta}}\left(\frac{k}{k+1}\right)^{n-l}, \quad l=m, m+1,
$$

if and only if

$$
\bar{F}(x)=e^{-\alpha(x / \theta)^{\beta}}, \quad 0<x<\infty, \quad \alpha>0, \beta, \theta>0,
$$

where

$$
\xi(y)=e^{-\alpha(x / \theta)^{\beta}}
$$

Proof. From (1.2) and (1.1), we have

$$
\begin{aligned}
E\left[\xi\left(Y_{n}^{(k)}\right) \mid\left(Y_{m}^{(k)}\right)=x\right]= & \frac{k^{n-m}}{(n-m-1) !} \int_{x}^{\infty} e^{-\alpha(y / \theta)^{\beta}}[\ln \bar{F}(x)-\ln \bar{F}(y)]^{n-m-1} \\
& \times\left(\frac{\bar{F}(y)}{\bar{F}(x)}\right)^{k-1} \frac{f(y)}{\bar{F}(x)} d y
\end{aligned}
$$

By setting $u=\frac{\bar{F}(y)}{\bar{F}(x)}=\frac{e^{-\alpha(y / \theta)^{\beta}}}{e^{-\alpha(x / \theta)^{\beta}}}$ from (1.4) in (4.5), we have

$$
E\left[\xi\left(Y_{n}^{(k)}\right) \mid\left(Y_{m}^{(k)}\right)=x\right]=\frac{k^{n-m}}{(n-m-1) !} e^{-\alpha(x / \theta)^{\beta}} \int_{0}^{1}(-\ln u)^{n-m-1} u^{k} d u
$$

We have Gradshteyn and Ryzhik ([5], p-551)

$$
\int_{0}^{1}(-\ln x)^{\mu-1} x^{v-1} d x=\frac{\Gamma \mu}{v^{\mu}}, \quad \mu>0, \quad v>0 .
$$

On using (4.7) in (4.6), we have the result given in (4.4). 
To prove sufficient part, we have

$$
\begin{aligned}
\frac{k^{n-m}}{(n-m-1) !} \int_{x}^{\infty} e^{-\alpha(x / \theta)^{\beta}}[\ln F(x)-\ln F(y)]^{n-m-1} \\
\quad \times[\bar{F}(y)]^{k-1} f(y) d y=[\bar{F}(x)]^{k} g_{n \mid m}(x),
\end{aligned}
$$

where

$$
g_{n \mid m}(x)=e^{-\alpha(x / \theta)^{\beta}}\left(\frac{k}{k+1}\right)^{n-m} .
$$

Differentiating (4.8) both sides with respect to $x$, we get

$$
\begin{aligned}
& -\frac{k^{n-m} f(x)}{\bar{F}(x)(n-m-2) !} \int_{x}^{\infty} e^{-\alpha(y / \theta)^{\beta}}[\ln F(x)-\ln F(y)]^{n-m-2} \\
& \quad \times[\bar{F}(y)]^{k-1} f(y) d y=g_{n \mid m}^{\prime}(x)[\bar{F}(x)]^{k}-k g_{n \mid m}(x)[\bar{F}(x)]^{k-1} f(x),
\end{aligned}
$$

or

$$
-k g_{n \mid m+1}(x)[\bar{F}(x)]^{k-1} f(x)=g_{n \mid m}^{\prime}(x)[\bar{F}(x)]^{k}-k g_{n \mid m}(x)[\bar{F}(x)]^{k-1} f(x) .
$$

Therefore,

$$
\begin{aligned}
\frac{f(x)}{\bar{F}(x)} & =-\frac{g_{n \mid m}^{\prime}(x)}{k\left[g_{n \mid m+1}(x)-g_{n \mid m}(x)\right]} \\
& =\frac{\alpha \beta}{\theta}(x / \theta)^{\beta-1},
\end{aligned}
$$

where

$$
\begin{gathered}
g_{n \mid m}^{\prime}(x)=-\frac{\alpha \beta}{\theta}\left(\frac{x}{\theta}\right)^{\beta-1} e^{-\alpha(x / \theta)^{\beta}}\left(\frac{k}{k+1}\right)^{n-m}, \\
g_{n \mid m+1}(x)-g_{n \mid m}(x)=\frac{1}{k} e^{-\alpha(x / \theta)^{\beta}}\left(\frac{k}{k+1}\right)^{n-m} .
\end{gathered}
$$

Integrating both sides of (4.9) with respect to $x$ between $(0, y)$, the sufficiency part is proved. 


\section{References}

[1] M. Ahsanullah, Record Statistics, Nova Science Publishers, New York, 1995.

[2] K. N. Chandler, The distribution and frequency of record values, J. Roy. Statist. Soc. Ser. B 14 (1952), 220-228.

[3] W. Dziubdziela and B. Kopociński, Limiting properties of the $k$-th record value, Appl. Math. (Warsaw) 15 (1976), 187-190.

[4] K. Danielak and M. Z. Raqab, Sharp bounds for expectations of $k$-th record increments, Aust. N. Z. J. Stat. 46 (2004), 665-673.

[5] I. S. Gradshteyn and I. M. Ryzhik, Table of Integrals Series and Products, Academic Press, New York, 2007.

[6] Z. Grudzien, Characterization of distribution of time limits in record statistics as well as distributions and moments of linear record statistics from the samples of random numbers, Praca Doktorska UMCS Lublin (1982).

[7] Z. Grudzień and D. Szynal, Characterization of continuous distributions via moments of $k$-th record values with random indices, J. Appl. Statist. Sci. 5 (1997), 259-266.

[8] J. S. Hwang and G. D. Lin, On a generalized moments problem II, Proc. Amer. Math. Soc. 91 (1984), 577-580.

[9] U. Kamps, A Concept of Generalized Order Statistics, B. G. Teubner Stuttgart, Germany, 1995.

[10] A. H. Khan, R. U. Khan and M. Yaqub, Characterization of continuous distributions through conditional expectation of function of generalized order statistics, J. Appl. Probab. Stat. 1 (2006), 115-131.

[11] M. A. Khan and R. U. Khan, $k$-th upper record values from modified Weibull distribution and characterization, Int. J. Comp. Theo. Stat. 3 (2016), 75-80.

[12] R. U. Khan, M. A. Khan and M. A. R. Khan, Relations for moments of generalized record values from additive Weibull distribution and associated inference, Stat. Optim. Inf. Comput. 5 (2017), 127-136.

[13] R. U. Khan, A. Kulshrestha and M. A. Khan, Relations for moments of $k$-th record values from exponential-Weibull lifetime distribution and a characterization, J. Egyptian Math. Soc. 23 (2015), 558-562.

[14] S. Minimol and P. Yageen Thomos, On some properties of Makeham distribution using generalized record values and its characterization, Braz. J. Probab. Stat. 27 (2013), 487-501.

[15] S. Minimol and P. Yageen Thomos, On characterization of Gompertz distribution by properties of generalized record values, J. Stat. Theory Appl. 13 (2014), 38-45.

[16] S. Nasiru and A. Luguterah, The new Weibull-Pareto distribution, Pak. J. Stat. Oper. Res. 11 (2015), 103-114. 
[17] P. Pawlas and D. Szynal, Recurrence relations for single and product moments of $k$-th record values from Weibull distributions and a characterization, J. Appl. Statist. Sci. 10 (2000), 17-26.

[18] P. Pawlas and D. Szynal, Relations for single and product moments of $k$-th record values from exponential and Gumble distributions, J. Appl. Statist. Sci. 7 (1998), 53-62.

[19] P. Pawlas and D. Szynal, Recurrence relations for single and product moments of $k$-th record values from Pareto generalized Pareto and Burr distributions, Comm. Statist. Theory Methods 28 (1999), 1699-1709. 